https://helda.helsinki.fi

Minimum viable metapopulation size.

Hanski, llkka

1996

American Naturalist. 147(4): 527-541. 1996.

http://hdl.handle.net/1975/7570

Downloaded from Helda, University of Helsinki institutional repository.

This is an electronic reprint of the original article.

This reprint may differ from the original in pagination and typographic detail.

Please cite the original version. 


\title{
MINIMUM VIABLE METAPOPULATION SIZE
}

\author{
Ilkka Hanski, ${ }^{1}$ Atte Moilanen, ${ }^{1}$ and Mats Gyllenberg ${ }^{2}$ \\ 'Department of Ecology and Systematics, Division of Population Biology, P.O. Box 17 ip. \\ Rautatiekatu 13), University of Helsinki, FIN-00014 Helsinki, Finland; and 'Department of \\ Mathematics, University of Turku, FIN-20500 Turku, Finland
}

Submitted January 25, 1995; Revised August 1, 1995; Accepted August 16, 1995

\begin{abstract}
We define the minimum viable metapopulation (MVM) size as the minimum number of interacting local populations necessary for long-term persistence of a metapopulation in a balance between local extinctions and recolonizations. The minimum amount of suitable habitat (MASH) is defined as the minimum density (or number) of suitable habitat patches necessary for metapopulation persistence. Levins's metapopulation model suggests that MASH can be estimated by the fraction of empty patches in a network in which the metapopulation occurs at a stochastic steady state. We discuss three reasons why this rule of thumb is likely to give an underestimate, and possibly a severe underestimate, of MASH: the rescue effect, colonizationextinction stochasticity, and nonequilibrium (transient) metapopulation dynamics. The assumption that metapopulations occur at a steady state, common to many models, may be frequently violated because of the high rate of habitat loss and fragmentation in many landscapes. Scores of rare and endangered species may already be "living dead," committed to extinction because extinction is the equilibrium toward which their metapopulations are moving in the present fragmented landscapes. To conserve these species we should reverse the process of habitat loss and fragmentation.
\end{abstract}

Many empirical studies have shown that the expected lifetime of a population increases with its current size (Williamson 1981; Diamond 1984; Schoener and Spiller 1987). Provided that the environment does not change greatly (unrealistic as this assumption may be), very large populations are expected to last for so long that no conservation measures are called for, whereas very small populations are likely to become extinct rapidly. The minimum viable population (MVP) size is intended to be an estimate of the minimum number of individuals in a population that has a good chance of surviving for some relatively long period of time, for instance, a 95\% chance of surviving for at least $100 \mathrm{yr}$ (Soulé 1980). Although difficult to apply in practice (Soulé 1987; Lande 1988), MVP is a useful concept in highlighting the need for a quantitative analysis of the risk of population extinction.

In the case of metapopulations consisting entirely of small and, hence, extinction-prone local populations (Hanski and Gilpin 1991), a somewhat analogous concept, minimum viable metapopulation (MVM) size, may be defined as the minimum number of interacting local populations necessary for the long-term persistence of the metapopulation. In addition, it is useful to consider the minimum amount of suitable habitat (MASH) necessary for metapopulation persis- 
tence, because not all suitable habitat is generally simultaneously occupied by a metapopulation persisting in a balance between local extinctions and recolonizations. In the metapopulation context, one has to ask questions about extinction due to permanent loss of habitat (the "declining-population paradigm" in Caughley 1994), whereas the concept of MVP is often applied to (small) local populations threatened by extinction for reasons other than systematic environmental change (the "small-population paradigm" in Caughley 1994).

The classical metapopulation scenario (Levins 1969) assumes a large network of small habitat patches inhabited by a set of local populations with a substantial risk of stochastic local extinction. The Levins metapopulation survives in a balance between local extinctions and recolonizations of empty but suitable habitat patches. Denoting by $p$ the fraction of currently occupied patches, the wellknown Levins model (Levins 1969, 1970) gives the instantaneous rate of change in $p$ as

$$
\frac{d p}{d t}=m p(1-p)-e p,
$$

where the parameters $m$ and $e$ set the rates of colonization and extinction, respectively.

To model habitat loss, assume that a fraction $1-h$ of the patches is permanently destroyed. The colonization rate thereby becomes lowered, because the density of empty but suitable patches available for colonization is decreased from $1-p$ to $h-p$, and the model becomes (May 1991; Nee and May 1992; Lawton et al. 1994; Nee 1994; Moilanen and Hanski 1995)

$$
\frac{d p}{d t}=m p(h-p)-e p .
$$

At equilibrium, the fraction of empty patches (out of all patches, including the destroyed ones) is given by

$$
h-p^{*}=\frac{e}{m},
$$

where $p^{*}$ is the fraction of occupied patches at steady state. Thus, the fraction (and the number in some given area) of empty patches out of all patches remains constant as long as the metapopulation does not become extinct, which happens when $h<e / m$ (fig. $1 A$ ). This is a seemingly very useful result, because it leads to the following rule of thumb, which we name here the Levins rule: A necessary and sufficient condition for metapopulation survival is that the remaining number of habitat patches following a reduction in patch number exceeds the number of empty but suitable patches prior to patch destruction.

The Levins rule is attractive because it gives an estimate of MASH from the very limited information of the number of empty patches in a landscape in which the metapopulation survives; no detailed knowledge of metapopulation dynamics is required (Nee 1994). In practice, though, the Levins rule is liable to yield an underestimate, and possibly a severe underestimate, of the critical patch number. One should be aware of this bias so as not to endorse the preservation of too 


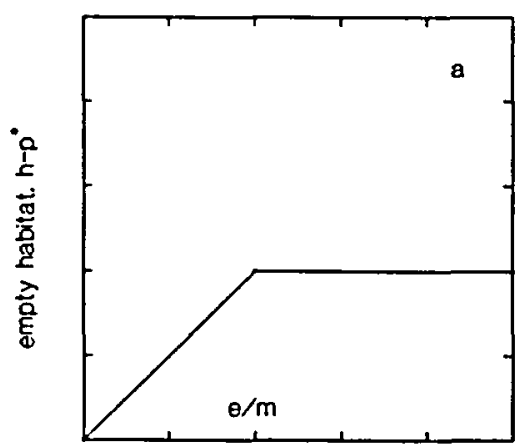

amount of habltat. $\mathrm{n}$

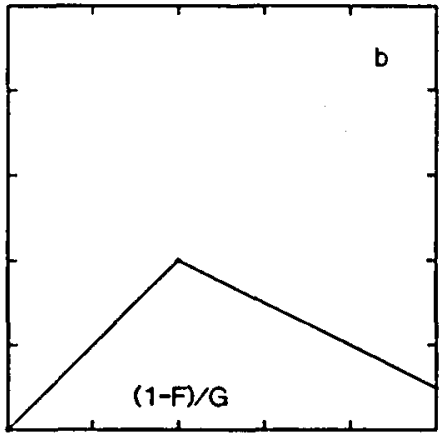

amount of habltat. $h$

FIG. 1.-A, A schematic illustration of the Levins rule (eq. [3]) that gives the relationship between the fraction of empty patches at equilibrium $\left(h-p^{*}\right)$ and the fraction of suitabl: patches. $B$, The analogous result for the discrete-time model (eq. [5]) with rescue effect $(r=1)$.

little habitat. Here, we describe and discuss three reasons why the Levins rule will probably underestimate MASH: the rescue effect, colonization-extinction stochasticity, and nonequilibrium metapopulation dynamics. By examining these three processes, we highlight partial answers to the twin questions about MVM and MASH.

\section{THE RESCUE EFFECT}

The Levins model (eq. [1]) gives the instantaneous rate of change in the fraction of occupied habitat patches. For mathematical convenience, changes in the sizes of local populations (local dynamics) are ignored, and newly established populations are assumed to grow instantaneously to the local carrying capacity. Therefore, the Levins model best applies to metapopulations inhabiting relatively small and isolated habitat fragments, because then local dynamics occur relatively fast in comparison with metapopulation dynamics. Although there are good examples of such metapopulations (Hanski and Hammond 1995; Hanski et al. 1995a), the assumption of distinct timescales of local and metapopulation dynamics is violated by the majority of metapopulations that have been studied by ecologists.

To see why this matters, let us consider the following discrete-time version of the Levins model:

$$
p_{t+1}=F p_{t}+G p_{t}\left[h-(1-r) p_{t}-r F p_{t}\right],
$$

where $p_{t}$ is the fraction of occupied patches at time $t, F$ is the probability of a population surviving one time interval, $G$ is the probability of recolonization given that $p_{\ell}=1$, and $r$ is a parameter giving the strength of the rescue effect (Brown and Kodric-Brown 1977). By the rescue effect we refer here to decreased probability of extinction between time instants $t$ and $t+1$ due to "simultaneous" recolonization before $t+1$. We envision that, in practice, the rescue effect is 


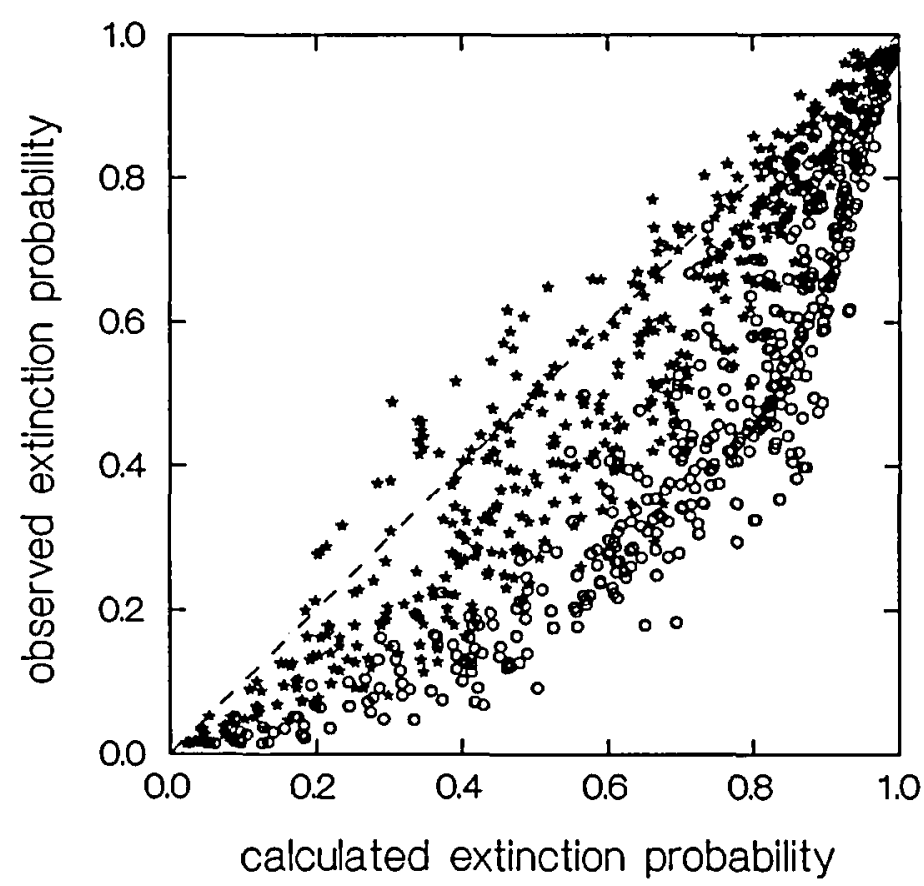

F1G. 2.-The relationships between the observed probability of local extinction ( $E_{\text {obs }}$; vertical axis) and the calculated extinction probability without the rescue effect ( $E_{\text {int }} ;$ circles) and the product $E_{\text {int }}(1-C)$ (asterisks, where $C$ is the probability of recolonization of an empty patch). Note that $E_{\text {obs }} \approx E_{\text {int }}(1-C)$, which suggests that $r \approx 1$ in equation (4). These results are for the Glanville fritillary, Melitaea cinxia, living in a large network of habitat patches (Hanski et al. 1995a; 1. Hanski, unpublished data). The broken line indicates equality of the extinction rates.

generated by immigration increasing population sizes and thereby decreasing the risk of extinction. The term in brackets in equation (4) is the fraction of patches available for colonization, including the fraction that went extinct between $t$ and $t+1\left([1-F] p_{t}\right)$ but may become rescued as specified by the value of $r$. If there is no rescue $(r=0)$, the model given by equation (4) has the same dynamic properties as the Levins model (eq. [1]), and the Levins rule remains valid. However, whenever the rate of migration is high, it may be assumed, as a first approximation, that the probability of rescue in this model equals the probability of establishment of a new population in a patch that was empty at time $t$, in which case $r=1$.

Figure 2 gives an empirical example of the rescue effect from a well-studied butterfly metapopulation (Hanski et al. 1994, 1995a, 1996). The observed extinction probability, $E_{\text {obs }}$, was estimated for each extant population in $1993(n=524)$ by fitting a logistic regression model to data on population extinction from 1993 until 1994 (256 extinctions). The independent variables were population size in 1993, patch area, a measure of the numbers of butterflies in the neighboring populations ( $S_{1993}$, calculated as explained in eq. [5] of Hanski 1994a), and a measure of the change in the sizes of the neighboring populations between 1993 
and $1994\left(\log \left[S_{1994} / S_{1993}\right]\right)$. The observed extinction probability includes any' possible rescue effect. An estimate of the extinction probability without the rescue effect, $E_{\text {int }}$, was then calculated by setting, in the logistic regression, the number of butterflies in the neighboring populations $\left(S_{1993}\right)$ to zero. The probability $E_{\text {int }}$ is often much greater than the observed probability $E_{\text {obs }}$ (fig. 2). Finally, we note that the observed extinction probability $E_{\text {obs }}$ is roughly matched by $E_{\text {int }}(1-C)$ (fig. 2), where $C$ is the estimated probability of recolonization of an empty patch (estimated with another logistic regression). These results suggest that, in this system, $r \approx 1$.

If $r=1$, the Levins rule (eq. [3]) is replaced by

$$
h-p^{*}=\frac{1-F}{F}\left(\frac{1}{G}-h\right) \text {. }
$$

Clearly, the fraction of empty patches is not independent of $h$ but grows with decreasing $h$ (fig. $1 B$ ). As in the Levins model, the metapopulation goes extinct if $h<(1-F) / G$. The important point is that, unlike in the Levins model, the fraction of empty patches cannot be used to estimate the amount of habitat necessary for metapopulation survival.

We emphasize that the difference between equations (3) and (5) is no: due to a difference between a continuous and discrete model. In a continuous-time structured metapopulation model (Gyllenberg and Hanski 1992), which includes an explicit description of local dynamics and migration and therefore mechanistically accounts for the rescue effect, the fraction of empty patches increases with increasing habitat destruction, just as in the above discrete-time model (M. Gyllenberg and I. Hanski, unpublished manuscript). The difference between equations (3) and (5) is due to the rescue effect, which cannot be naturally incorporated into the continuous-time Levins model (but see Hanski 1991).

We draw the following conclusion. The Levins rule remains valid for metapopulations with low turnover rate and a very low rate of habitat destruction, because then the rescue effect is of little importance and the metapopulation has time to closely track the changing environment in spite of a low colonization rate (recall that the Levins rule applies to a metapopulation at equilibrium). Unfortunately, this is not the kind of scenario that conservation biologists are presently worried about. In reality, turnover rate is often high, the rescue effect should not be ignored (fig. 2), and the rate of environmental change is fast (see Nonequilibrium Metapopulation Dynamics below). The discrete-time model and the result (eq. [5]) derived from it apply to these situations, with the caveat that the metapopulation may not turn over fast enough to justify the equilibrium assumption. We return to this point below.

\section{COLONIZATION-EXTINCTION STOCHASTICITY}

The models discussed so far are meant to apply to large networks of habitat patches with many local populations. In practice, the number of patches and local populations is often small, and a metapopulation that is predicted to persist by 
equations (1) and (4) may nonetheless go extinct owing to chance variation in the number of extant populations. Such colonization-extinction stochasticity (Hanski 1991) is analogous to demographic stochasticity (May 1973) in the dynamics of small local populations.

Gurney and Nisbet (1978; summarized in Nisbet and Gurney 1982) have analyzed a stochastic version of the Levins model. Their analysis yielded the following approximation for the expected time to metapopulation extinction, $T_{M}$,

$$
T_{\mathrm{M}}=T_{\mathrm{L}} e^{H p^{* 2} / 2\left(1-p^{*}\right)},
$$

where $T_{\mathrm{L}}$ is the expected time to local extinction and $H$ is the number of suitable habitat patches. If one defines long-term metapopulation persistence as $T_{\mathrm{M}}$ exceeding 100 times $T_{\mathrm{L}}$, equation (6) leads to the following condition for a reasonably large value for $H$ (Gurney and Nisbet 1978):

$$
p^{*} \sqrt{H} \geq 3 \text {. }
$$

For example, if there are 50 habitat patches, equation (7) says that the colonization and extinction rates must be such that $p^{*}>0.42$ for the metapopulation to persist for longer than 100 times $T_{\mathrm{L}}$.

These results were derived from a stochastic Levins model with identical habitat patches, no spatial structure, and no rescue effect. We shall explore below, numerically, the validity of equation (7) in a spatially realistic metapopulation model, the incidence function model (Hanski 1994a), in which these restrictions can be relaxed. The incidence function model relates the stationary probability of patch occupancy to patch size, to isolation from existing populations, and possibly to other patch attributes. We first assumed a hypothetical network of 100 equally large patches located randomly in a square area. Colonization probability was modeled either as an exponential or sigmoid function of the expected number of migrants arriving at a patch. The former assumption corresponds more closely with the assumptions of the Levins model, but the latter is often more realistic (Hanski 1994a). We assumed the rescue effect, as in equation (4), and assumed that migration was either of island type as in the Levins model or spatially restricted. In a second set of simulations, we used the incidence function model to iterate the dynamics of the Glanville fritillary, Melitaea cinxia, in real networks of habitat patches using parameter values estimated from field data (Hanski et al. 1995a, 1996). To examine the effect of the number of patches on metapopulation survival, we created smaller patch networks by deleting patches in the periphery of the more extensive network, thus keeping patch density relatively constant (details of these simulations are described in the appendix).

The results in figure $3 A$ and $B$ demonstrate that equation (7) gives a reasonably good approximation in the case of exponential colonization probability, regardless of whether migration is of island type or restricted to nearby patches. The spatial extent of migration makes no great difference, at equilibrium, when all patches have statistically similar degrees of connection to other patches (this would be violated if patches were strongly aggregated). With sigmoid colonization probability there is more variation in the results (fig. $3 \mathrm{C}$ ), apparently because with sigmoid colonization probability there is somewhat more variation in the fraction of occu- 

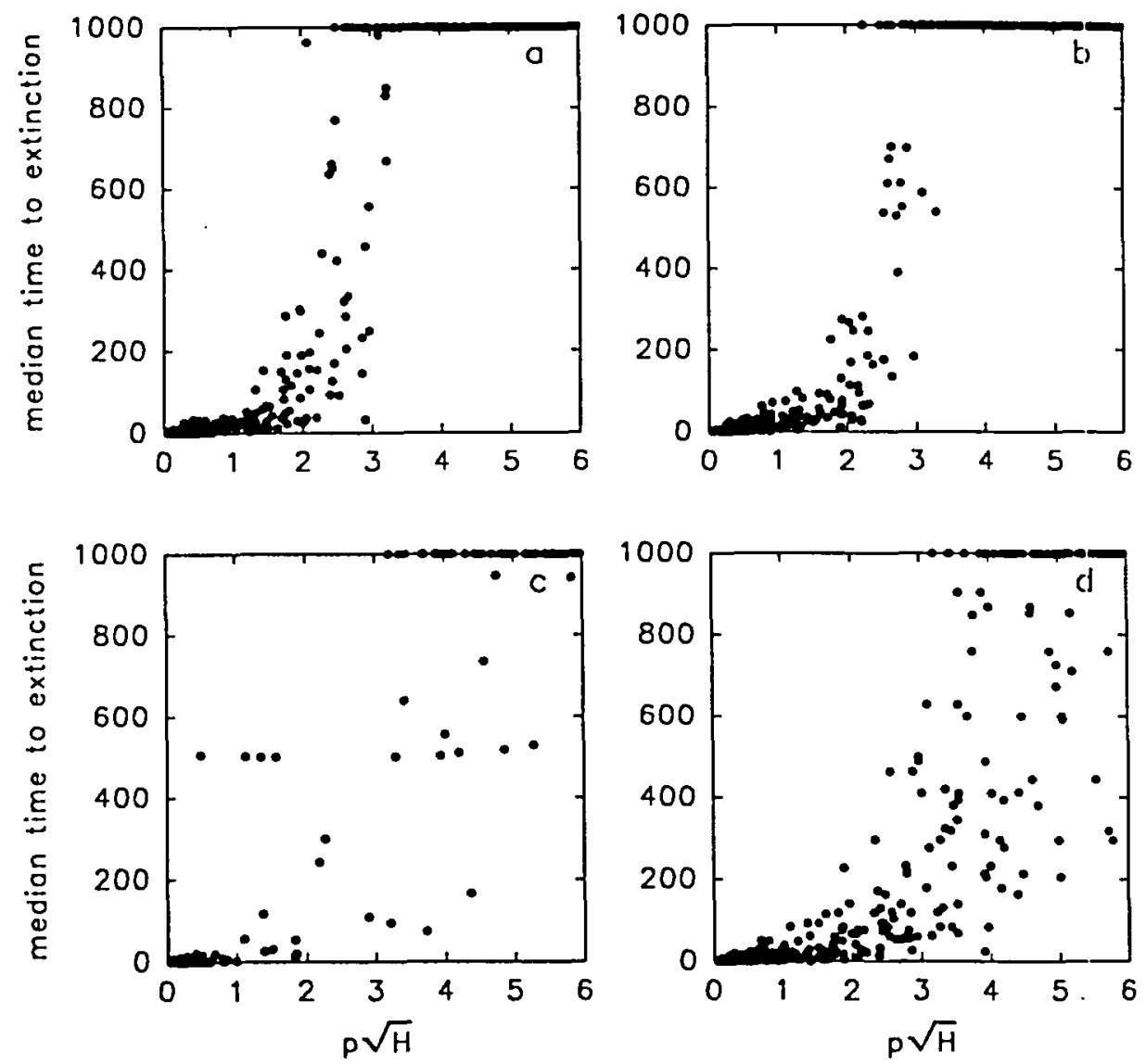

FIG. 3. - The relationship between the median time to metapopulation extinction and the product $p^{*} \sqrt{H}$ in the model results described in the text and in the appendix (median $T_{\mathrm{L}}$ $=3.3$ in these examples). $A$, Hypothetical network with island-type migration (not dependent on distance). $B$, Hypothetical network with distance-dependent migration and patches equal in size to those in $A$. Both $A$ and $B$ assume exponentially increasing probability of colonization with the number of immigrants arriving at an empty patch. $C$, Hypothetical network with sigmoid colonization probability (eq. [3] in Hanski 1994a) and restricted migration. $D$, Hypothetical network with spatially correlated environmental stochasticity (completely correlated stochastic variation in the sizes of local populations).

pied patches at stochastic steady state. Variance in $p$ is greatly increased by adding regional stochasticity (spatially correlated environmental stochasticity), which substantially shortens metapopulation lifetime (fig. $3 D$ ). (Increased variance in $p$ also increases the error with which its value is determined from ernpirical data [Hanski et al. 1996].)

In the case of the butterfly example, with large variance in patch areas (Hanski et al. 1995a), metapopulations tended to survive for a long time when, roughly, $p^{*} \sqrt{H}>2$ (fig. $4 A$ ). The reason for the enhanced survival in comparison with the examples in figure 3 appears to be the variance in patch areas and, hence, in 

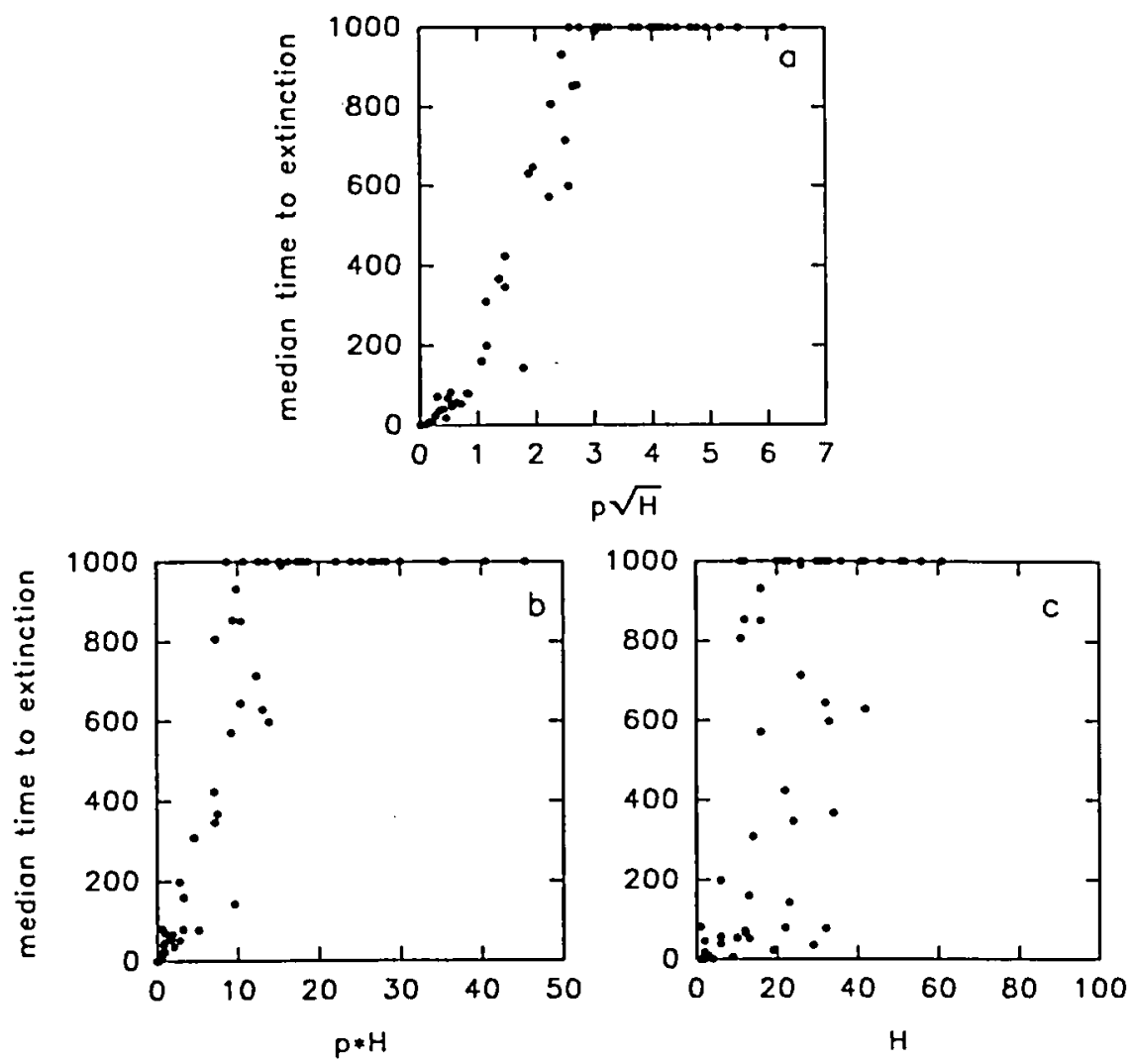

FIG. 4. $-A$, The relationship between median time to metapopulation extinction and the product $p^{*} \sqrt{H}$ in model results using networks of real habitat patches inhabited by the Glanville fritillary, Melitaea cinxia. See the appendix for details. $B$, The median time to metapopulation extinction as a function of the expected number of extant populations. $C$, The median time to metapopulation extinction as a function of the number of habitat patches in the network.

local population sizes: metapopulation lifetime is increased by the low risk of extinction of the largest local populations (extinction probability was here $\approx 0.3$ for median-sized patches compared with $<0.01$ for the largest ones).

It should be noted that the characteristics of the species and the patch network as reflected in the value of $p^{*}$ are combined with the size of the patch network in equation (7). Rearranging equation (7) in the form $p^{*} H>3 \sqrt{H}$ suggests that MVM is roughly given, in this model, by $3 \sqrt{H}$. Because MVM is a function of $H$, the theory says that MVM and MASH cannot be evaluated independently. In practice, though, the number of extant populations at equilibrium $\left(p^{*} H\right)$ should be a much better predictor of metapopulation persistence than the number of habitat patches, because differences in the properties of patch networks (other than $H$ ) and in the properties of species are integrated in the value of $p^{*}$. This view is supported by the results in figure $4 B$ and $C$ for the butterfly example. To 
take another example, consider modifying a particular patch network by keeping patch number constant but reducing patch density by increasing their average isolation. Assuming that migration is spatially restricted, reduced patch density will lower colonization rate and thereby decrease the value of $p^{*}$ and, hence, reduce the chances of metapopulation survival for a given $H$. Even a very large number of habitat patches is not sufficient for metapopulation persistence if these patches are spread thinly across a large area. Much of the scatter in figure $4 C$ can be explained by variation in the average isolation and average size of the patches in the 14 networks that were used to generate these results (see the appendix).

\section{NONEQUILIBRIUM METAPOPULATION DYNAMICS}

The Levins rule assumes that the metapopulation that we observe is at at stochastic steady state. This may not be so. Habitat fragmentation has occurred rapidly in many landscapes, and it is likely that many metapopulations are presently approaching a new equilibrium from above, with "too many" patches currently occupied (Hanski 1994b; Tilman et al. 1994). If one estimates the minimum patch number necessary for metapopulation persistence using observations from such nonequilibrium metapopulations, one will necessarily underestimate MASH.

We illustrate nonequilibrium metapopulation dynamics with an example from our research on the dynamics of the Glanville fritillary, Melitaea cinxia, on the Åland islands in Finland (Hanski et al. 1994, 1995a, 1996). Frank Hering (personal communication) has recently surveyed an area of approximately $25 \mathrm{~km}^{2}$ with 42 extant habitat patches (dry meadows) suitable for this butterfly. Using 15-20-yrold aerial photographs and ground surveys of the vegetation, Hering arrived at the result shown in figure 5 about the extent of suitable habitat approximately 20 yr ago and today. During this period, the total area of suitable habitat declined to $33 \%$ of its original extent, and the number of patches declined from 55 to 42 , largely owing to decreased grazing pressure on the habitat patches.

In the absence of more detailed information, we used the following assumptions about the decline in patch areas during the past $20 \mathrm{yr}$. For each patch that lost some area (fig. 5), we assumed that the loss occurred linearly over a period of $T$ years, where $T$ is a random variate uniformly distributed between 1 and $20 \mathrm{yr}$. We then calculated with the parameterized incidence function model (Hanski 1994a; Hanski et al. 1996) the fraction of occupied patches $p$ during and following the 20-yr period of habitat destruction, as well as the equilibrium value of $p$. The difference between the two reflects the delay in metapopulation dynamics in a declining patch network.

The result in figure $6 \mathrm{~A}$ suggests that this butterfly metapopulation has tracked the amount of suitable habitat with only a small delay. The inferred changes in metapopulation size are largely due to fast turnover in small populations. However, one should not draw the conclusion that the same result would necessarily apply to all scenarios of habitat loss even in this species. The following example makes this point forcefully. Let us assume that each of the present patches would lose a further $50 \%$ of its area in another $20 \mathrm{yr}$. Figure $6 \mathrm{~B}$ shows that such further 


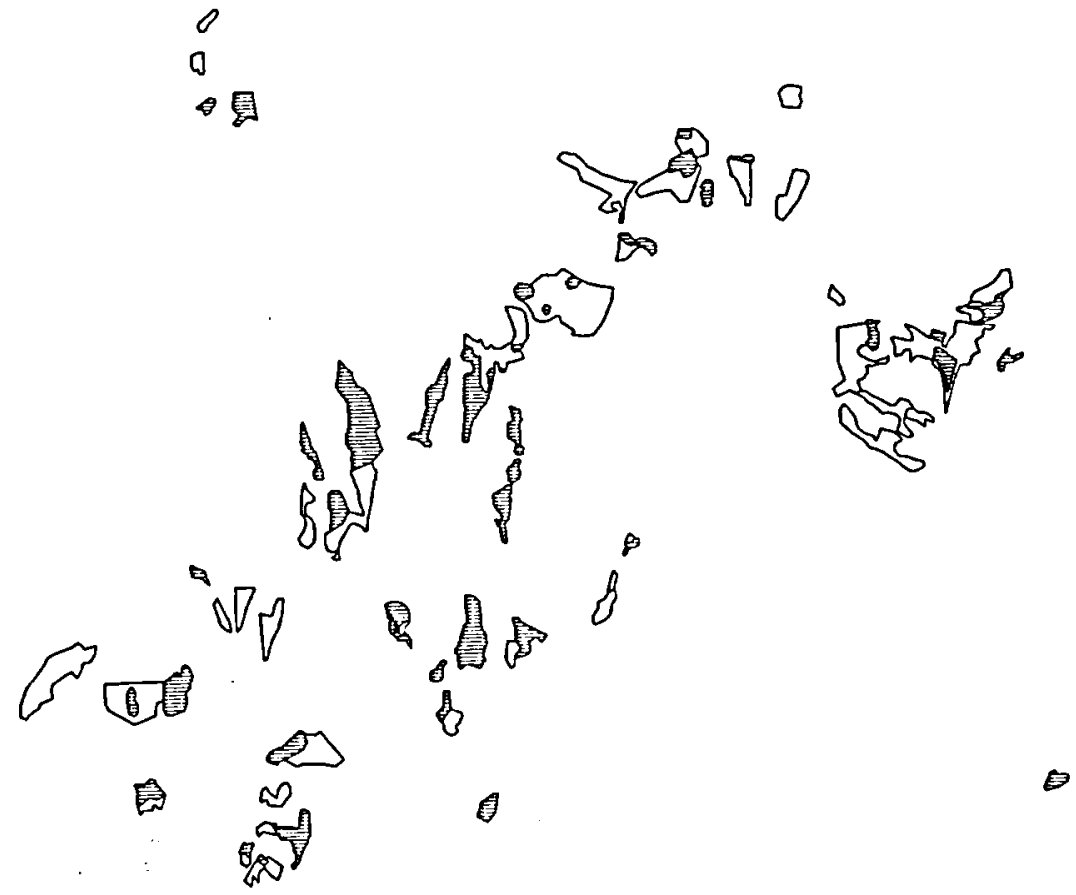

FIg. 5.-A map of the study area (ca. $25 \mathrm{~km}^{2}$ ) on Åland Island, Finland, showing the presumed extent of the habitat patches suitable for the Glanville fritillary, Melitaea cinxia. approximately $20 \mathrm{yr}$ ago and today (shaded) (F. Hering, personal communication).

loss of habitat area would soon lead to a patch network smaller than MASH for this species. But in this case, the actual metapopulation extinction is predicted to take tens or even hundreds of years (fig. $6 B$ ). The inevitable decline to extinction may become temporarily halted for long periods, with the number of occupied patches fluctuating without any obvious trend (fig. 7). The final decline to extinction is slow because the last populations to go are typically the largest ones with the smallest risk of extinction. The delay would be smaller if the dynamics were greatly affected by regional stochasticity (spatially correlated environmental stochasticity).

\section{DISCUSSION AND CONCLUSIONS}

We have described three reasons why the Levins rule may give an underestimate of the minimum amount of suitable habitat necessary for long-term metapopulation persistence. The level of underestimation may be severe, and we conclude that, although the rule has some heuristic value, it should not be used to justify further loss of habitat.

The Levins rule fails in metapopulations with substantial migration among local populations leading to strong rescue effect. More complex metapopulation models incorporating the effect of migration on local dynamics illustrate another possible 

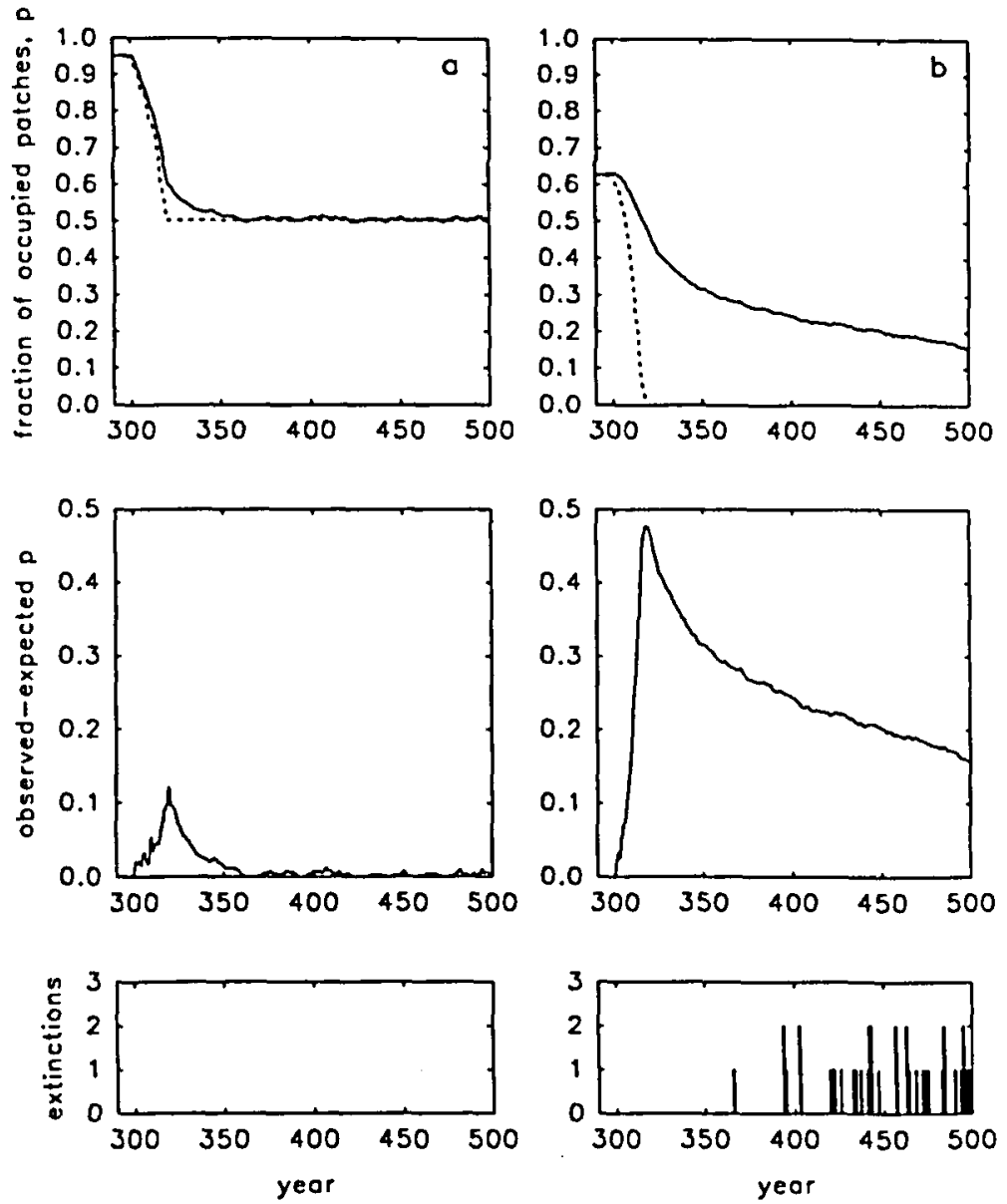

FIG. 6.-A, Metapopulation size of the Glanville fritillary, Melitaea cinxia, as measured by the fraction of occupied patches in the landscape shown in fig. 5 . The results were obtained with the incidence function model (Hanski 1994a) with parameter values estimated from data collected in 1993. The model iteration was started by assuming the patch network $20 \mathrm{yr}$ ago (fig. 5). During a period of $20 \mathrm{yr}$ (from year 300 to 320), the network was reduced to its present size (fig. 5) as described in the text. The broken line gives the equilib:ium metapopulation size, whereas the continuous line gives the expected metapopulation size in the declining network. The lines give the average value of $p$ in 200 replicate simulations. The middle panel gives the difference between the actual and equilibrium metapopulation sizes. and the lower panel gives the numbers of metapopulation extinctions at different points in time (no extinctions in this case). $B$, The metapopulation size as in $A$ but starting with the current patch network (fig. 5) and halving the area of each patch in $20 \mathrm{yr}$. Note that the equilibrium metapopulation size drops to zero, but it takes tens of years for most metapopulations to reach the equilibrium (extinction). 


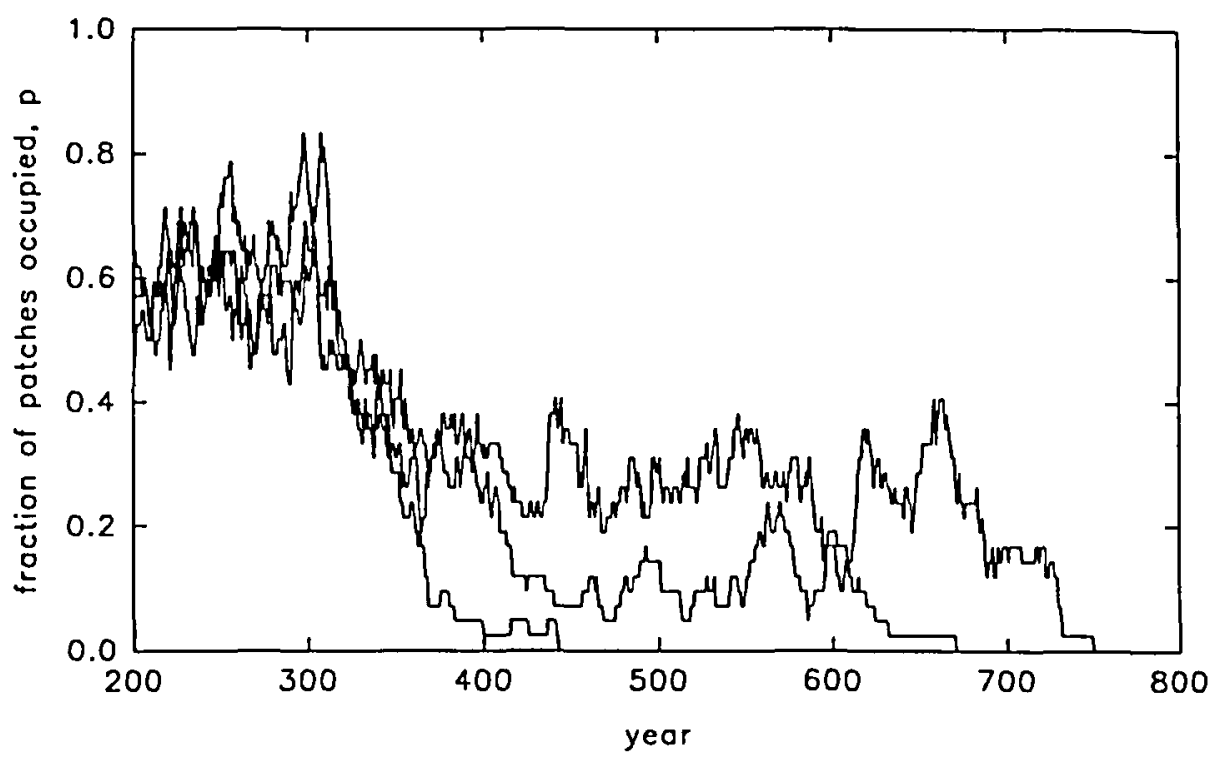

FIG. 7. -Three examples of actual metapopulation trajectories from the simulations described in fig. $6 B$. Note the magnitude of oscillations and how two of the three metapopulations settled down to a low value of $p$ for a long time before eventual extinction. Regional stochasticity would increase the amplitude of oscillations and shorten the time to extinction.

dynamic outcome with implications for conservation and management. Assuming, realistically, that there is some cost to migration (increased mortality) and propagule size-dependent (sigmoid) probability of colonization of empty patches, the models demonstrate the possibility of alternative stable equilibria, one of which is metapopulation extinction (Hanski 1985; Hastings 1991; Gyllenberg and Hanski 1992; Hanski and Zhang 1992). A metapopulation with alternative stable equilibria may suddenly collapse to extinction from a large size, with most patches occupied. Therefore, not all common species are necessarily safe from extinction even in only slightly degraded environments. Results for the Glanville fritillary, which we have used repeatedly in this article, strongly suggest the occurrence of alternative stable equilibria in metapopulation dynamics (Hanski et al. 1995b).

Stochastic models make the point that metapopulations consisting of a small number of local populations, each with a high risk of extinction, are not likely to survive for a long time. Although it would be unwise to draw a general conclusion from the butterfly example in figure 4, we tentatively suggest that for metapopulations living in networks of small habitat patches, comparable to the system inhabited by the Glanville fritillary (Hanski et al. 1995a, 1996), MVM is of the order of 10 extant populations (fig. $4 B$ ), and the number of suitable patches in the network should exceed roughly 20 for likely long-term persistence, although depending on the kind of network, 20 patches may not be sufficient (fig. $4 C$ ). Empirical results (reviewed in Thomas and Hanski 1996) for other species of butterflies are in broad agreement with these suggestions. 
We conclude by emphasizing three other points. The number of extant populations $Q$ (where $Q=p^{*} H$ ) is perhaps the most useful and practical measure to assess the viability of metapopulations in stationary environments. In the example in figure $4, Q$ predicts metapopulation persistence as well as the quantity $p^{*} \sqrt{H}$, as suggested by theory. In addition, $Q$ has the advantage of being easier to measure in practice, as there is no need to count the suitable but empty habitat patches; $Q$ is a superior measure to the number of suitable patches (fig. $4 B, C$ ), because $Q$ accounts for variation in the spatial configuration and size distribution of habitat patches. The catch, however, is that assessing metapopulation viability by the number of extant populations assumes that the metapopulation is at a stochastic steady state, which may not be the case.

Our second point is that, although these results can legitimately be used to justify the need for networks of a minimum of 15-20 well-connected patches (fig. $4 C$ ), one should not rush to the conclusion that when this requirement cannot be met, there is no rational basis for protecting the remaining few populations and habitat patches and undertaking other management measures. The theoretical result refers to long-term survival of a metapopulation in relation to the expected lifetime of local populations. Local populations themselves may last for tens of years (for well-documented butterfly examples, see Warren 1992), and a metapopulation expected to survive for a shorter period of time than "long-term" might nonetheless survive for many decades or even longer. Practically all landscapes change much faster than that, and indeed, the assumption that metapopulations occur at equilibrium is dubious for many, although not all, species. Especially where habitat has been recently destroyed, there is the possibility that the metapopulation has not had time to reach the new equilibrium, which may be extinction. Just like an old tree left without its pollinators and dispersal agents (Janzen and Martin 1982), a metapopulation may occur in a fragmented landscape, not because the landscape structure is adequate for long-term persistence but by virtue of the slowness of the decline to extinction. This sort of scenario is usually envisioned in the case of faunal collapse on relict habitats at a large spatial scale, following, for instance, postglacial climate change and complete isolation of populations (Harrison 1991), but as the example in figures 5-7 illustrates, the same sort of events may be rampant all around us. This leads to our third ancl most disturbing conclusion for conservation: many rare and endangered species may already be committed to extinction, unless the loss and fragmentation of their habitat is reversed.

\section{APPENDIX}

\section{Design of the Simulation Model}

The results in figure 3 were obtained by assuming a hypothetical patch network, which was generated by randomly placing 100 patches in a square $2 \times 2 \mathrm{~km}^{2}$. All patch $6: \mathrm{s}$ were $272 \mathrm{~m}^{2}$ in size, which equals the median patch size in the Melitaea cinxia metapopulation (described in Hanski et al. 1995a) used in figure 4. Smaller patch networks $(n=4,7, \ldots$, 100 patches) were created by removing the patches with the greatest distance to the center of the original network, thus keeping patch density relatively constant. Metapopulation dynamics were iterated with the incidence function model (Hanski 1994a) with the follow- 
ing field-estimated parameter values: $x=0.952, e=0.01$, and $\alpha=-1.0$ (Hanski et al. 1996). Different $p^{*}$ values for a particular patch network were generated by changing the value of parameter $y$, which determines colonization probability (Hanski 1994a).

Twenty replicate simulation runs for each combination of $n$ and $y$ were conducted as follows. All iterations were started with all patches occupied. The results for the first 500 generations were omitted to allow the metapopulation to reach a stochastic steady state. The observed $p^{*}$ value was calculated as the median of the $p$ values during the next 500 generations. To prevent premature metapopulation extinction, each patch had a small extra probability of colonization (1\% per year) during the first 1,000 generations. Following the determination of the $p^{*}$ value, the external colonization probability was set to zero, and the model was iterated until the metapopulation became extinct or 1,000 generations had elapsed. Finally, the median time to extinction and the average $p^{*}$ value were calculated for the 20 replicates.

The results in figure 4 were obtained in the same manner as those in figure 3, but with a different set of patches. Here 14 large patch networks occupied by the Glanville fritillary, Melitaea cinxia (Hanski et al. 1995a), were iterated with field-estimated parameter values (table 1 in Hanski et al. 1996). Smaller networks were generated by removing 10 patches at a time from the periphery of the network until there were fewer than 10 patches left. The parameter $y$ was kept constant (4.04; Hanski et al. 1996).

\section{LITERATURE CITED}

Brown, J. H., and A. Kodric-Brown. 1977. Turnover rates in insular biogeography: effect of immigration on extinction. Ecology 58:445-449.

Caughley, G. 1994. Directions in conservation biology. Journal of Animal Ecology 63:215-244.

Diamond, J. M. 1984. "Normal" extinctions of isolated populations. Pages 191-246 in M. H. Nitecki, ed. Extinctions. University of Chicago Press, Chicago.

Gurney, W. S. C., and R. M. Nisbet. 1978. Single-species population fluctuations in patchy environments. American Naturalist 112:1075-1090.

Gyllenberg. M., and I. Hanski. 1992. Single-species metapopulation dynamics: a structured model. Theoretical Population Biology 42:35-61.

Hanski, 1. 1985. Single-species spatial dynamics may contribute to long-term rarity and commonness. Ecology 66:335-343.

1991. Single-species metapopulation dynamics: concepts, models and observations. Pages 17-38 in M. E. Gilpin and I. Hanski, eds. Metapopulation dynamics. Academic Press, London.

1994a. A practical model of metapopulation dynamics. Journal of Animal Ecology 63:151-162.

1994b. Spatial scale, patchiness and movement on land. Philosophical Transactions of the Royal Society of London B, Biological Sciences 343:19-25.

Hanski, I., and M. E. Gilpin. 1991. Metapopulation dynamics: brief history and conceptual domain. Pages 3-16 in M. E. Gilpin and I. Hanski, eds. Metapopulation dynamics. Academic Press, London.

Hanski, I., and P. Hammond. 1995. Biodiversity in boreal forests. Trends in Ecology \& Evolution 10:5-6.

Hanski, I., and D.-Y. Zhang. 1993. Migration, metapopulation dynamics and fugitive co-existence. Journal of Theoretical Biology 163:491-504.

Hanski, 1., M. Kuussaari, and M. Nieminen. 1994. Metapopulation structure and migration in the butterfly Melitaea cinxia. Ecology 75:747-762.

Hanski, I., T. Pakkala, M. Kuussaari, and G. Lei. 1995a. Metapopulation persistence of an endangered butterfly in a fragmented landscape. Oikos 72:21-28.

Hanski, I., J. Pöyry, T. Pakkala, and M. Kuussaari. 1995b. Multiple equilibria in metapopulation dynamics. Nature (London) 377:618-621.

Hanski, I., A. Moilanen, T. Pakkala, and M. Kuussaari. 1996. Metapopulation persistence of an endangered butterfly: a test of the quantitative incidence function model. Conservation Biology, in press. 
Harrison, S. 1991. Local extinction in a metapopulation context: an empirical evaluation. Pages 73-88 in M. Gilpin and 1 . Hanski, eds. Metapopulation dynamics. Academic Press, London.

Hastings, A. 1991. Structured models of metapopulation dynamics. Pages 57-71 in M. E. Gilpin and I. Hanski, eds. Metapopulation dynamics. Academic Press, London.

Janzen, D. H., and P. S. Martin. 1982. Neotropical anachronisms: the fruits the Gomphotheres ate. Science (Washington, D.C.) 215:19-27.

Lande, R. 1988. Genetics and demography in biological conservation. Science (Washington, D.C.) 241:1455-1460.

Lawton, J. H., S. Nee, A. J. Letcher, and P. H. Harvey. 1994. Animal distributions: patterns and processes. Pages 41-58 in P. J. Edwards, R. M. May, and N. R. Webb, eds. Large-scale ecology and conservation biology. Blackwell, Oxford.

Levins, R. 1969. Some demographic consequences of environmental heterogeneity for biclogical control. Bulletin of the Entomological Society of America 15:237-240.

1970. Extinction. Pages 77-107 in M. Gesternhaber, ed. Some mathematical problems in biology. American Mathematical Society, Providence, R.I.

May, R. M. 1973. Complexity and stability in model ecosystems. Princeton University Press, Princeton, N.J.

1991. The role of ecological theory in planning reintroduction of endangered species. Siymposia of the Zoological Society of London 62:145-163.

Moilanen, A., and I. Hanski. 1995. Habitat destruction and coexistence of competitors in a spatially realistic metapopulation model. Journal of Animal Ecology 61:141-144.

Nee, S. 1994. How populations persist. Nature 367:123-124.

Nee, S., and R. M. May. 1992. Dynamics of metapopulations: habitat destruction and competitive coexistence. Journal of Animal Ecology 61:37-40.

Nisbet, R. M., and W. S. C. Gurney. 1982. Modelling fluctuating populations. Wiley, New York.

Schoener, T. W., and D. A. Spiller. 1987. High population persistence in a system with high turnover. Nature (London) 330:474-477.

Soule, M. E. 1980. Thresholds for survival: maintaining fitness and evolutionary potential. Pages 111-124 in M. E. Soule and B. A. Wilcox, eds. Conservation biology: an evolutionaryecological perspective. Sinauer, Sunderland, Mass.

- 1987. Viable populations for conservation. Cambridge University Press, Cambridge.

Thomas, C. D., and I. Hanski. 1996. Butterfly metapopulations. In I. Hanski and M. Gilpin, eds. Metapopulation dynamics: ecology, genetics and evolution. Academic Press, Loniton (in press).

Tilman, D., R. M. May, C. L. Lehman, and M. A. Nowak. 1994. Habitat destruction and the extinction debt. Nature (London) 371:65-66.

Warren, M. S. 1992. The conservation of British butterflies. Pages 246-274 in R. L. H. Dennis, ed. The ecology of butterflies in Britain. Oxford University Press, Oxford.

Williamson, M. 1981. Island populations. Oxford University Press, Oxford. 